Descargo de responsabilidad: las opiniones expresadas en los manuscritos son responsabilidad exclusiva de los autores. No necesariamente reflejan las opiniones de la editorial ni la de sus miembros.

1

\title{
Integración laboral como estrategia de empleo para jóvenes con discapacidad intelectual
}

Labor integration as an employment strategy for young people with intellectual disabilities

Jennie Karina Esteban Gutierrez

jekarina04@gmail.com

Recibido: 12/10/2021

Publicado: 20/01/2022

https://orcid.org/0000-0001-6762-6022

Elly Evangelina del Rosario Estrada González

ellyestrada87@gmail.com

Filiación institucional de los autores

Maestría en Neurociencia con énfasis en Neurocognición

Universidad de San Carlos de Guatemala
Ana Josefina Fajardo Estrada

mapaesgt@gmail.com

https://orcid.org/0000-0002-3158-2694

Rosa María Linares Reyes

rosalinares82@hotmail.com

https://orcid.org/0000-0003-4542-9698

\section{Referencia}

Esteban Gutierrez, J. K., del Rosario Estrada González, E. E., Fajardo Estrada, A. J., \& Linares Reyes, R. M. (2022). Integración laboral como estrategia de empleo para jóvenes con discapacidad intelectual. Revista Académica Sociedad Del Conocimiento Cunzac, 2(1), 33-40. DOI: https://doi.org/10.46780/sociedadcunzac.v2i1.10

\section{Resumen}

OBJETIVO: conocer sobre la realidad laboral de las personas con discapacidad intelectual en la ciudad de Guatemala y describir la integración laboral como una estrategia para su acceso a la fuerza de trabajo. MÉTODO: se realizó un estudio descriptivo transversal en 110 padres, familiares o tutores de jóvenes o adultos con discapacidad intelectual. RESULTADOS: se demostró que el rango etario más frecuente de los individuos con dicha condición fue de 18 a 25 años de edad con un $72 \%(\mathrm{~N}=79)$, encontrando el género masculino como predominante en un $82 \%(\mathrm{~N}=89)$, se evidenció en el $77 \%$ de los casos $(\mathrm{N}=84)$ que las personas con discapacidad intelectual no tienen trabajo. CONCLUSIÓN: Ios adultos jóvenes con discapacidad intelectual de la ciudad de Guatemala carecen de opciones para ingresar al mundo laboral y es necesario modificar los paradigmas actuales para lograr su integración sociolaboral.

\section{Palabras clave}

discapacidad intelectual, inclusión laboral, integración laboral 


\section{Abstract}

OBJECTIVE: learn about the labor reality of people with intellectual disabilities in Guatemala City and describe labor integration as a strategy for their access to the workforce. METHOD: a descriptive cross-sectional study was carried out in 110 parents, relatives or guardians of young people or adults with intellectual disabilities. RESULTS: it was shown that the most frequent age range of individuals with this condition was from 18 to 25 years of age with $72 \%(\mathrm{~N}=79)$, finding the male gender as predominant in $82 \%(\mathrm{~N}=89)$, It was evidenced in $77 \%$ of the cases $(\mathrm{N}=84)$ that people with intellectual disability do not have a job. CONCLUSION: young adults with intellectual disability in Guatemala City lack options to enter the world of work and it is necessary to modify the current paradigms to achieve their socio-labor integration.

\section{Keywords}

intellectual disability, labor inclusion, social integration

\section{Introducción}

La discapacidad es una condición que no depende del género, clase social, nacionalidad ni de otras características demográficas, en Guatemala, según la presentación de los resultados de la Segunda Encuesta Nacional de Discapacidad (ENDIS 2016) el Consejo Nacional Para la Atención de las Personas con Discapacidad (CONADI), el Fondo de las Naciones Unidas para la Infancia (UNICEF) y el Instituto Nacional de Estadística (INE), el 10.2\% de la población presenta algún tipo de discapacidad, es decir, cerca de 1 millón 600 mil personas. Este es un dato que amerita atención ya que la mayoría de personas con discapacidad son discriminadas y excluidas de la sociedad que se refleja en la dificultad de encontrar desde la infancia una educación de calidad adaptada a sus necesidades propias y posteriormente al mundo laboral en la edad adulta.

En años posteriores, Ramírez (2020) establece que la Organización Panamericana de la Salud (OPS) en su Informe Mundial sobre Discapacidad indica que el $15 \%$ de la población presenta algún tipo de discapacidad y sus manifestaciones, lo que incrementa a 2 millones 400 mil personas en toda la República. Confirma que muchos de ellos no tienen acceso a la salud o educación y tan sólo un $2 \%$ tiene un empleo estable. Este incremento se debe en su mayoría a la falta de nutrición y acceso a la salud pública en áreas urbanas como rurales.

Por su lado, la Biblioteca Nacional de los Estados Unidos (2021), manifiesta que la discapacidad intelectual afecta entre 1 al 3\% de la población global. Con base a lo anterior, se calcula que en la ciudad de Guatemala, un aproximado de 24,000 personas presentan Discapacidad Intelectual, 2,880 de ellos en edad productiva de 18 a 49 años y que solamente alrededor de 58 personas tienen un empleo estable.

A través de los años, se han pronunciado diferentes instituciones gubernamentales y privadas que se esfuerzan para apoyar a esta población mediante diferentes programas. Entre ellas, el Instituto Neurológico de Guatemala (mediante el Programa de Atención Integral para la Inclusión Laboral (PAIIL), la Fundación Margarita Tejada (con su programa dirigido específicamente para personas con Síndrome de Down), el Centro de Capacitación Ocupacional (CCO) de la 
Secretaría de Bienestar Social del Gobierno de la República con su programa público que está dirigido a adolescentes con discapacidad intelectual provenientes de familias de escasos recursos económicos, avalado por el Instituto Técnico de Capacitación (INTECAP). Asimismo, es necesario mencionar que también la Fundación Kinal, que consiste en un centro educativo privado, no lucrativo, dirigido a la formación técnica profesional de jóvenes y adultos, realiza inclusión siempre y cuando el estudiante haya cursado hasta el sexto primaria, sea capaz de leer y escribir.

El tema de la inclusión o integración laboral ha sido un desafío para diversos países alrededor del mundo. En Zaragoza, España, Pallisera (2010) reconoce que esta transición se trata de un proceso sumamente complejo puesto que amerita evolucionar a la edad adulta dejando atrás los programas educativos inclusivos. La escuela o instituciones de apoyo juegan un papel importante en este proceso para ser un puente que facilite la inclusión laboral. Sin embargo, se presentan muchos obstáculos para que una persona con discapacidad intelectual pueda lograr autonomía laboral debido a que las estrategias implementadas no lo facilitan, adicional a la falta de control e incumplimiento en los aspectos establecidos en la ley con relación a la inclusión de dichas personas en el ámbito laboral. A través de su análisis, Pallisera sugiere que se debe enfatizar en el reconocimiento las políticas sociales y educativas que favorecen dicha transición a la vida laboral activa, así como proponer un cambio de rol en los educadores para que dirijan sus estrategias educativas para una real inclusión laboral construyendo proyectos enfocados en las necesidades de esta población.

Como se puede observar, todas las instituciones están enfocadas a capacitar a los jóvenes con discapacidad intelectual para optar por la tan anhelada inclusión laboral de un mundo ideal. Todas las personas con discapacidad intelectual que son capacitadas para laborar, necesitan encontrar un empleo adecuado a sus habilidades. Lamentablemente, en nuestro país, el $98 \%$ no tienen éxito para conseguirlo, teniendo que quedarse en casa o, en el mejor de los escenarios, emprender individualmente. En la mayoría de estos casos, claudican por no tener la estructura empresarial que los apoya, no teniendo otra opción que seguir siendo totalmente dependientes de sus padres o en su ausencia, de sus hermanos u otro familiar que se haga cargo de ellos.

El concepto de inclusión se refiere a brindar a las personas con discapacidad las mismas oportunidades que a las personas neurotípicas. No obstante, no se toma en cuenta que ellos tienen una forma diferente de aprender, desempeñarse y relacionarse con los demás; en muchas ocasiones, si las personas que trabajan con ellos no son educadas y sensibilizadas al respecto, pueden no comprender su manera de comportarse y mal interpretarlo causando fricción entre los mismos empleados y perjudicando el ambiente laboral. Además, su nivel de productividad no será el mismo al de un empleado neurotípico si el empleador no conoce y comprende su perfil de funcionalidad, campo que generalmente es abordado exclusivamente por profesionales de la conducta humana o terapeutas ocupacionales.

La inclusión se refiere a incluir a la persona con discapacidad en el grupo neurotípico haciendo el esfuerzo de "acomodar" lo más posible el ambiente para que ellos puedan convivir. Sin embargo, la convivencia no es lo mismo que realización personal y productividad. Se deben cambiar paradigmas y analizar lo verdaderamente importante fuera de los límites de acción de las entidades ya existentes. 
En Málaga, España, Negri y Leiva (2016), reconocen que es importante promover nuevas estrategias para facilitar la inserción laboral con amplitud de opciones adaptadas a los intereses y diversas necesidades de las personas con "Diversidad Funcional Intelectual", término utilizado en Europa para referirse a personas con Discapacidad Intelectual. Partiendo de una educación inclusiva, los jóvenes necesitan ser también integrados a la sociedad para radicar su discriminación y fomentar la solidaridad. Una de las herramientas con más eficiencia en la integración laboral a jóvenes con discapacidad intelectual en España, es el trabajo con apoyo social, donde los jóvenes se pueden capacitar en talleres ocupacionales al mismo tiempo que brindan sus servicios a entidades con fines sociales. La integración laboral se refiere entonces a brindarles la oportunidad de acceder al mundo laboral en un ambiente controlado y desarrollado exclusivamente para ellos, cubriendo sus necesidades y respetando su forma de desempeño, lo que provocará que tengan las mismas oportunidades que las demás personas. Escudero (2021) establece que, mediante la Fundación Mosaico Down en México, se apoya a personas con Síndrome de Down y a personas con discapacidad intelectual desde su infancia por medio de estrategias de inclusión educativa, buscando generar conocimiento y preparando a los jóvenes y adultos para una vida independiente. Ellos lograr su objetivo mediante una carrera técnica en gastronomía (que abarca desde su educación, capacitación laboral e inclusión social) con el fin de ser integrados a sus empresas propias de servicio de catering y restaurante. Vale la pena mencionar que éste es un proyecto que ha iniciado este mismo año.

En nuestro país, un proyecto similar se ha venido desarrollando durante los últimos cinco años, en la ciudad capital, por medio del Centro Terapéutico Mapaes que desde su fase inicial, trabaja el área terapéutica con niños y adolescentes con desafíos o alteraciones en su desarrollo, aprendizaje y/o conducta, así como en su segunda fase, que se enfoca al trabajo con jóvenes con Discapacidad Intelectual mediante de un programa terapéutico y ocupacional desarrollado para lograr su autonomía personal tanto como su condición lo permita.

El proyecto de emprendimiento para la integración laboral "Kikilos" consiste en la tercera fase de este programa y sería pionero al establecer las bases de una nueva perspectiva para el acceso al mundo laboral para personas con discapacidad intelectual al no solamente proporcionar la debida capacitación en oficios, sino combinando técnicas terapéuticas y ocupacionales para mejorar tanto sus procesos cognitivos como su estadío emocional, se lograría la deseada integración mediante un modelo de negocios unificado.

\section{Material y métodos}

Se realizó un estudio descriptivo transversal en 110 padres, familiares o tutores de jóvenes o adultos con discapacidad intelectual durante el mes de septiembre en la Ciudad de Guatemala con el fin de conocer el rango etario, género, nivel de dependencia y estado laboral de las personas con discapacidad intelectual. El desarrollo de la investigación se realizó por medio de encuesta. 


\section{Resultados}

Tabla 1. Características Sociodemográficas de Personas con Discapacidad Intelectual.

\begin{tabular}{ccc}
\hline & Cantidad & Porcentaje \\
\hline Edad & & \\
\hline 18 a 25 años & 80 & 73 \\
\hline 26 a 35 años & 21 & 19 \\
\hline Mayores de 35 años & 9 & 8 \\
\hline Género & & \\
\hline Masculino & 90 & 82 \\
\hline Femenino & 20 & 18 \\
\hline
\end{tabular}

Fuente: elaboración propia.

La edad como característica sociodemográfica se tabula según rango etario identificando entre los 18 a 25 años con 79 casos que representan el $73 \%$, 26 a 35 años con 20 casos reflejando el $19 \%$ y mayores de 35 años con 9 casos que denotan $8 \%$ de la población estudio. El género de mayor frecuencia fue el masculino con 90 casos que representan el $82 \%$ de la población en relación con el género femenino con 20 casos que refleja el $18 \%$.

Tabla 2. Nivel de Severidad de las Personas con Discapacidad Intelectual.

\begin{tabular}{ccc}
\hline & Cantidad & Porcentaje \\
\hline Nivel & & \\
\hline Leve & 16 & 15 \\
\hline Moderada & 50 & 45 \\
\hline Severa & 44 & 40 \\
\hline
\end{tabular}

Fuente: elaboración propia.

El nivel de severidad que prevaleció fue la Discapacidad Intelectual Moderada abarcando 50 casos reflejados en el $45 \%$ de la población, seguida por la severa con 44 casos (40\%) y la leve con 16 casos (15\%). 
Tabla 3. Dependencia Económica de las Personas con Discapacidad Intelectual.

\begin{tabular}{ccc}
\hline & Cantidad & Porcentaje \\
\hline $\begin{array}{c}\text { Paga parcialmente sus } \\
\text { gastos }\end{array}$ & 2 & 2 \\
\hline $\begin{array}{c}\text { Depende de sus padres } \\
\text { Depende de sus } \\
\text { hermanos }\end{array}$ & 73 & 66 \\
\hline Depende de otro familiar & 23 & 21 \\
\hline
\end{tabular}

Fuente: elaboración propia.

El $66 \%$ de las personas con discapacidad depende económicamente de sus padres. El $21 \%$ lo hace de sus hermanos, el $11 \%$ de otros familiares y solamente el $2 \%$ trabaja y paga parcialmente sus gastos. Es decir, el $100 \%$ de los familiares de las personas con discapacidad están conscientes de su dependencia económica, lo que les produce altos niveles de preocupación debido a la incertidumbre que les agobia al pensar qué va a suceder con sus hijos o familiares, cuando ellos tengan que faltar (fallecimiento).

\section{Discusión}

Según los resultados obtenidos mediante el estudio transversal, se puede enfatizar la falta de opciones que las personas con discapacidad intelectual afrontan para lograr tener ese derecho de ser útiles a la sociedad, como cualquier otra persona.

Por años, las instituciones del país han desarrollado un modelo de inclusión laboral que ha logrado el resultado esperado para personas con discapacidad, específicamente la intelectual, en un bajo porcentaje (aproximadamente $2 \%$ ) apoyando solamente a alrededor de 58 familias en toda la ciudad capital. Todas estas entidades se enfocan a capacitar al recurso humano, pero no así a buscar eficientemente ofertas de empleo para ellos. Algunas empresas inician a incluir a personas mayormente con discapacidad física (no videntes, no oyentes, problemas motores) en su recurso humano, pero las opciones para la integración laboral de personas con discapacidad intelectual en nuestro país son limitadas y se encuentran en una etapa embrionaria, debido a que recién comienzan a elaborar políticas públicas que sirven como la base legal para que las empresas inicien a crear conciencia para brindarles una oportunidad de empleo.

Es determinante una nueva mirada acerca de la discapacidad, una visión alejada del déficit y centrada en las fortalezas que demuestran los trabajadores con esta condición. Es necesario encontrar nuevas estrategias con el fin de conseguirles un empleo digno y de calidad acorde a sus conocimientos y habilidades. Según Pegalajar (2015), mediante su experiencia en el 
proyecto "Capacitas" en Murcia, España, la inclusión sociolaboral de jóvenes con discapacidad intelectual favoreció una mejora de la calidad de vida en el ámbito de la autonomía, socialización, desarrollo personal, autoestima, autoconcepto y un aumento en el desarrollo de sus capacidades a nivel cualitativo y cuantitativo.

El proyecto "Kikilos" pretende llegar a ser esa nueva opción para la integración sociolaboral de esta población con una estructura unificada y estandarizada para poder crear un mayor número de oportunidades laborales.

\section{Referencias}

Biblioteca Nacional de Medicina de Estados Unidos (2021). Discapacidad Intelectual. Medline Plus https://medlineplus.gov/spanish/ency/article/001523.htm

CONADI (2016). Presentación de los Resultados de la II Encuesta Nacional de Discapacidad https://conadi.gob.gt/web/2017/03/21/presentacion-de-resultados-de-la-encuesta-nacional-de-discapacidad/

Escudero Y. (2021). Inclusión Laboral de Personas con Síndrome de Down. Revista Virtual Síndrome de Down 2021. https://www. down21.org/revista-virtual/1785-revista-virtual-sindrome-de-down-2021/revista-virtual-enero-2021-n-236/3525-inclusion-laboral-de-personascon-sindrome-de-down.html

Negri, M.I. y Leiva, J.J. (2016). El papel de la formación profesional en la inclusión socio-laboral de los jóvenes con Diversidad Funcional Intelectual. Revista de Educación Inclusiva. 9(3). https://revistaeducacioninclusiva.es/index.php/REl/article/view/250/244

Pallisera, M. (2011). La inclusión laboral y social de los jóvenes con discapacidad intelectual. El papel de la escuela Revista Interuniversitaria de Formación de Profesorado. https:// dugi-doc.udg.edu/bitstream/handle/10256/9612/InclusionLaboralSocial.pdf?sequence=1

Pegalajar, M. (2015). La inclusión sociolaboral de jóvenes con discapacidad intelectual: una experiencia a partir del programa "Capacitas". Revista de Educación Inclusiva. 8(1). https:// revistaeducacioninclusiva.es/index.php/REl/article/view/121/117

Ramírez, K. (2020). La vida de las Personas con Discapacidad Auditiva en Guatemala. Eco Resuena Gt. https://ecogtresuena.wordpress.com/2020/08/28/la-vida-de-las-personas-con-discapacidad-auditiva-en-guatemala/

\section{Sobre las autoras}

Grupo de investigadoras de la Maestría en Neurociencias con énfasis en Neurocognición, Universidad de San Carlos de Guatemala. 


\section{Financiamiento de la investigación}

Con recursos propios de los investigadores

\section{Declaración de intereses}

Declaran no tener ningún conflicto de intereses, que puedan haber influido en los resultados obtenidos o las interpretaciones propuestas.

\section{Declaración de consentimiento informado}

El estudio se realizó respetando el Código de ética y buenas prácticas editoriales de publicación.

\section{Derechos de uso}

Copyright@ 2022 por Jennie Karina Esteban Gutierrez, Elly Evangelina del Rosario Estrada González, Ana Josefina Fajardo Estrada y Rosa María Linares Reyes.

Este texto está protegido por una licencia Creative Commons 4.0

Este texto está protegido por la Licencia Creative Commons Atribución 4.0 Internacional.

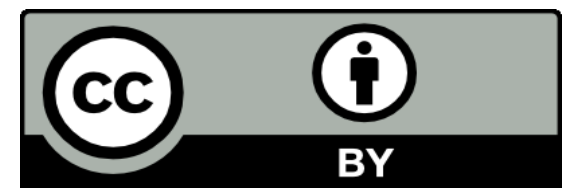

Este texto está protegido por una licencia

Creative Commons 4.0.

Es libre para compartir, copiar y redistribuir el material en cualquier medio o formato y adaptar el documento, remezclar, transformar y crear a partir del material para cualquier propósito, incluso comercialmente, siempre que cumpla la condición de atribución: debe reconocer el crédito de una obra de manera adecuada, proporcionar un enlace a la licencia, e indicar si se han realizado cambios. Puede hacerlo en cualquier forma razonable, pero no de forma tal que sugiera que tiene el apoyo del licenciante o lo recibe por el uso que hace. 